Original Article

\title{
Survival and Predictors of Mortality in Acute Kidney Injury Patients Treated with Sustained Low Efficiency Dialysis
}

Attaphong Phongphitakchai, ${ }^{1} M D$, Ussanee Boonsrirat, ${ }^{1} M D$

\begin{abstract}
Introduction: Sustained low efficiency dialysis (SLED) is an increasingly common treatment option for acute kidney injury (AKI) patients, but there are few studies examining the survival and predictive outcome of this therapy. The study aims to evaluate survival, pre-SLED predictors and complications associated with SLED. Materials and Methods: This was a retrospective cohort study of 91 patients with AKI treated with SLED in a tertiary hospital from January 2014 to August 2018. The primary outcomes were in-hospital and 30-day mortality. The secondary outcomes were the clinical and laboratory pre-SLED characteristics that were associated with survival and complication of SLED. Results: Median survival of AKI patients treated with SLED was 17 days and the 30-day mortality rate was $58 \%$. Pre-SLED serum levels of creatinine (adjusted HR $0.82,95 \%$ CI 0.71x0.94), albumin (adjusted HR 0.57, 95\% CI 0.4-0.81), potassium (adjusted HR 1.38, 95\% CI 1.1-1.73) and number of SLED (adjusted HR $0.95,95 \%$ CI 0.91-1) served as predictors of survival. Arrhythmia was found 3.3\% and intradialytic hypotension in $13.2 \%$ of patients. No patient had bleeding complications. Conclusions: Our study found similar in-hospital and 30-day mortality for AKI patients treated with SLED. High pre-SLED levels of serum albumin, creatinine and number of SLED were significantly associated with reduced risk of death and high pre-SLED serum potassium was associated with increased risk of death. These results indicate that SLED is safe treatment, with few haemorrhage and haemodynamic complications.
\end{abstract}

Key words: Acute kidney injury, Predictors, Sustained low efficiency dialysis, Survival

\section{Introduction}

Approximately one in ten patients admitted to an intensive care unit (ICU) develops acute kidney injury (AKI), an important complication of ICU patients, and requiring renal replacement therapy (RRT). ${ }^{1}$ It is well known that AKI contributes to mortality and chronic kidney diseases which result in health and economic burdens. ${ }^{2}$ Traditionally, there are 2 RRT modalities for ICU patients with AKI-continuous renal replacement therapy $(\mathrm{CRRT})^{3,4}$ and intermittent haemodialysis (IHD). However, these 2 modalities have shortcomings in their need of special devices, special nursing care and their high cost as well as the requirement for haemodynamic stabilility. Thus sustained low efficiency dialysis (SLED) is becoming more commonly used due to its ease of use and haemodynamic stability. ${ }^{5,6}$

SLED is a hybrid therapy which provides better haemodynamic tolerability, lower exposure to anticoagulation and shorter duration of therapy without changing the patient's clinical outcome or survival compared to CRRT. ${ }^{7}$ However, as this is a

${ }^{1}$ Division of Nephrology, Department of Internal Medicine, Faculty of Medicine, Prince of Songkla University, Hat Yai, Thailand.

Address for Correspondence: Dr Ussanee Boonsrirat, Division of Nephrology, Department of Internal Medicine, Faculty of Medicine, Prince of Songkla University, 15 Kanchanavanit Road, Hat Yai, Songkhla 90110, Thailand

Email: ussaneeboonsrirat@yahoo.com 
relatively new therapy, there are few studies examining the details of clinical and laboratory responses to it, information that is essential to evaluate the potential outcome of a patient undergoing SLED or factors influencing their survival. ${ }^{6,8}$ Therefore, this study aimed to evaluate survival and potential pre-SLED predictors of survival, and assess haemorrhagic and haemodynamic complications associated with SLED.

\section{Materials and Methods}

A retrospective, cohort study was conducted in Songklanagarind hospital which is a university hospital in Southern Thailand from Januray 2014 to August 2018. AKI patients aged 15 years or above treated with SLED who met the AKI criteria cited in The Kidney Disease Improving Global Outcomes (KDIGO) guidelines 2012. Further inclusion crieria were those with a serum creatinine level rising more than $0.3 \mathrm{mg} / \mathrm{dL}$ in 48 hours, urine output less than $0.5 \mathrm{ml} / \mathrm{kg} /$ hour for 6 hours or patients who were likely to suffer acute renal failure within 1 week of the serum creatinine level increasing 1.5-fold higher than the baseline serum creatinine. Those with end stage renal disease (ESRD) were excluded. The sample size was calculated based on one sample for estimating hazard in survival analysis considering hazard of $25 \%$ and $95 \%$ confidence intervals leading to a required sample size of 87 patients. The study was approved by the Ethics Committee of the Faculty of Medicine, Prince of Songkla University (REC 60-072-14-4)

Eligible patients were identified from the lists of patients undergoing haemodialysis in the ICU using SLED. The duration of one SLED session in our study was 6 to 12 hours with blood flow rate 150 to $250 \mathrm{ml} / \mathrm{min}$ and dialysate flow rate of 300 to $500 \mathrm{ml} / \mathrm{min}$. Normal saline or heparin was used as the anticoagulant. The medical records of eligible patients treated with SLED were reviewed for demographic and clinical characteristics, clinical and laboratory responses, and mortality.

The primary outcomes were in-hospital mortality and 30-day mortality. The secondary outcomes were the clinical and laboratory pre-SLED parameters that affect survival including severity score, mean arterial pressure and numbers of vasopressors before and after the SLED treatments and the SLED characteristics. Other variables were pre-SLED serum urea nitrogen, creatinine, potassium, calcium, phosphorus, magnesium and blood $\mathrm{pH}$. Independent variables included age, gender, cause of AKI, comorbidity, reason for ICU admission and mechanical ventilator uses were reviewed at the start of the SLED treatments. Bleeding complications after the SLED treatments were reviewed. Intradialytic hypotension was defined as an acute drop in systolic blood pressure below $80 \mathrm{mmHg}$ or more than $20 \%$ from baseline. Bleeding complication was defined as bleeding at the catheter exit site or systemic bleeding from anticoagulant use with SLED.

In-hospital mortality was analysed using the Kaplan-Meier method. The 30-day mortality rate of AKI treated with SLED was analysed descriptively. Predictors of survival were analysed by the Cox proportional hazards model. The laboratory responses were expressed as means and compared between pre-SLED, 24 hours and 48 hours post-SLED using the student paired t-test. The clinical responses were expressed as means and compared between pre-SLED and post-SLED using the student paired t-test. The SLED complications were expressed as numbers (percentages).

\section{Results}

During the study period, a total of 504 patients were admitted to the ICU for a cause requiring RRT and 91 of them met the additional inclusion criteria. Table 1 shows the baseline characteristic of all patients. Their ages ranged from 17 to 81 years and two-thirds were male. Approximately half of the AKIs were caused by acute tubular necrosis (ATN) and the most common comorbidity was sepsis. Almost all of the patients required mechanical ventilator and vasopressor. Of the 89 patients receiving vasopressor, 60 patients needed to increase dosage of norepinephrine and 10 patients needed to increase dosage of norepinephrine with use the additional dopamine. The mean dosage of norepinephrine (major vasopressor used) was $0.18 \mu \mathrm{g} / \mathrm{kg} / \mathrm{min}$ (ranges $0.02-0.2 \mu \mathrm{g} / \mathrm{kg} / \mathrm{min}$ ) and the mean dosage of dopamine (additional vasopressor used) was $5.2 \mu \mathrm{g} / \mathrm{kg} / \mathrm{min}$ (ranges 3.0-6.0 $\mu \mathrm{g} / \mathrm{kg}$ / $\mathrm{min})$. The cardiovascular system SOFA score 3 and 4 were 10 and 79 patients, repsectively. The eligble patients treated with SLED had high Sequential Organ Failure Assessment (SOFA, $13.3 \pm 3.4$ ) and Acute Physiology and Chronic Heath Evaluation scoring system version II (APACHE II, $29.3 \pm 5.8$ ) scores. Table 2 shows the SLED characteristics. The number of SLED treatments per patient ranged from 1 to 12 . The blood flow rate and dialysate flow rate ranged from 150 to $250 \mathrm{ml} / \mathrm{min}$ and 300 to $500 \mathrm{ml} / \mathrm{min}$ respectively. Ultrafiltration ranged from 0 to $2500 \mathrm{ml} / \mathrm{session}$ and the duration of SLED ranged from 8 to 12 hours. 
Table 1. Baseline characteristics of study patients

\begin{tabular}{|c|c|}
\hline Variables & $\begin{array}{l}\text { Number of patients } \\
\qquad(n=91)\end{array}$ \\
\hline Age (years), median (IQR) & $65(52,74)$ \\
\hline \multicolumn{2}{|l|}{ Gender, n (\%) } \\
\hline Male & $57(62.6)$ \\
\hline Female & $34(37.4)$ \\
\hline \multicolumn{2}{|l|}{ Cause of AKI } \\
\hline Pre-renal & $25(27.5)$ \\
\hline ATN & $41(45.1)$ \\
\hline $\mathrm{CIN}$ & $6(6.6)$ \\
\hline Post-renal & $1(1.1)$ \\
\hline Multiple causes & $18(19.8)$ \\
\hline \multicolumn{2}{|l|}{ Comorbidity, $n(\%)$} \\
\hline Sepsis & $51(56)$ \\
\hline Diabetes & $12(13.2)$ \\
\hline Heart disease & $19(20.9)$ \\
\hline Other & $9(9.9)$ \\
\hline \multicolumn{2}{|l|}{ Reason for ICU admission, $n(\%)$} \\
\hline Medical & $43(51.8)$ \\
\hline Surgical & $31(37.3)$ \\
\hline Cardiovascular & $8(9.6)$ \\
\hline Trauma & $1(1.2)$ \\
\hline Mechanical ventilator use, $\mathrm{n}(\%)$ & $84(92.3)$ \\
\hline Vasopressor use, n (\%) & $89(97.8)$ \\
\hline \multicolumn{2}{|l|}{ Laboratory (pre-SLED) } \\
\hline Serum urea nitrogen $(\mathrm{mg} / \mathrm{dl})$, mean $\pm \mathrm{SD}$ & $69.6(30.9)$ \\
\hline Serum creatinine $(\mathrm{mg} / \mathrm{dl})$, mean $\pm \mathrm{SD}$ & $4.9(2.8)$ \\
\hline SOFA score, mean \pm SD & $13.3(3.4)$ \\
\hline APACHE II score, mean \pm SD & $29.3(5.8)$ \\
\hline \multicolumn{2}{|c|}{$\begin{array}{l}\text { AKI: Acute Kidney Injury; APACHE II: Acute Physiology and } \\
\text { Chronic Heath Evaluation scoring system version II; ATN: Acute } \\
\text { Tubular Necrosis; CIN: Contrast Induced Nephropathy; ICU: Intensive } \\
\text { Careunit; IQR: Interquartile Range; SLED: Sustained Low Efficiency } \\
\text { Dialysis; SOFA: Sequential Organ Failure Assessment }\end{array}$} \\
\hline
\end{tabular}

Table 3 shows the laboratory responses after SLED treatment at 24 and 48 hours. Serum urea nitrogen, creatinine and potassium were significantly decreased after SLED at 24 and 48 hours. Acidosis was significantly improved after the SLED treatment at
Table 2. SLED characteristics

\begin{tabular}{lc}
\hline Variable & Mean \pm SD \\
\hline $\begin{array}{l}\text { Number of SLED treatments per patient, } \\
\text { median (IQR) }\end{array}$ & $5(2,8)$ \\
\hline Blood flow rate (millilitres/min) & $184.6(50.4)$ \\
Dialysate flow rate (millilitres/min) & $412.5(105.9)$ \\
Ultrafiltration (millilitres/day) & $1888(1658)$ \\
SLED duration per treatment (hours) & $10.41(1.73)$ \\
\hline
\end{tabular}

IQR: Interquartile Range; SLED: Sustained Low Efficiency Dialysis

both 24 and 48 hours. The data on the clinical responses and complications are presented in Table 4. Mean arterial pressure (MAP) and number of vasopressor used were not significantly different after SLED. Few instances of arrhythmia and intradialytic hypotension were observed, and there were no bleeding complications.

The 30-day mortality rate in AKI patients treated with SLED was $58 \%$. Figure 1 shows the Kaplan-Meier curve of the patients' survival rate. The median survival time was 17 days, with a maximum follow up of 210 days (7 months). During follow up, one third of the patients died within 7 days but the survival probability stablised after 60 days. Pre-SLED SOFA, serum creatinine, serum magnesium, serum potassium, serum albumin, number of SLEDs and MAP were the 7 predictors with $P$ value $<0.2$ and these were included in the first model of Cox proportional hazards regression model. Only 5 predictors remained significant in the final model (Table 5). For AKI patients treated with SLED, predictors of survival included pre-SLED serum creatinine (adjusted HR 0.82, 95\% CI 0.71-0.94), pre-SLED serum albumin (adjusted HR 0.57 , 95\% CI 0.4-0.81), pre-SLED serum potassium (adjusted HR 1.38, 95\% CI 1.1-1.73) and number of SLEDs (adjusted HR 0.95, 95\% CI 0.91-1), while pre-SLED SOFA (adjusted HR $1.08,95 \%$ CI $0.99-1.17$ ) was not significant. Patients with a high pre-SLED serum potassium had a 1.38 times higher risk for death. High pre-SLED serum creatinine, albumin and number of SLEDs had an $18 \%, 43 \%$ and $5 \%$ reduction in risk, respectively.

\section{Discussion}

A short median survival time of AKI patients treated with SLED was found and more than half of the patients died within 30 days of treatment. The pre- 
Table 3. Laboratory responses

\begin{tabular}{|c|c|c|c|c|c|}
\hline Variable & Pre-SLED & 24 hr Post-SLED & $P$-value & 48 hr Post-SLED & $P$-value \\
\hline $\begin{array}{l}\text { Serum urea nitrogen } \\
(\mathrm{mg} / \mathrm{dl}), \text { mean } \pm \mathrm{SD}\end{array}$ & $69.58(30.87)$ & $50.32(28.68)$ & $<0.001$ & $57.92(22.47)$ & $<0.001$ \\
\hline Serum creatinine (mg/dl) & $4.87(2.76)$ & $3.75(2.40)$ & $<0.001$ & $4.24(2.61)$ & 0.002 \\
\hline Serum potassium $(\mathrm{mmol} / \mathrm{L})$ & $4.43(0.97)$ & $4.02(0.88)$ & 0.001 & $3.99(0.60)$ & 0.001 \\
\hline Blood $\mathrm{pH}$ & $7.32(0.13)$ & $7.36(0.14)$ & 0.09 & $7.40(0.08)$ & $<0.001$ \\
\hline
\end{tabular}

SLED: Sustained Low Efficiency Dialysis

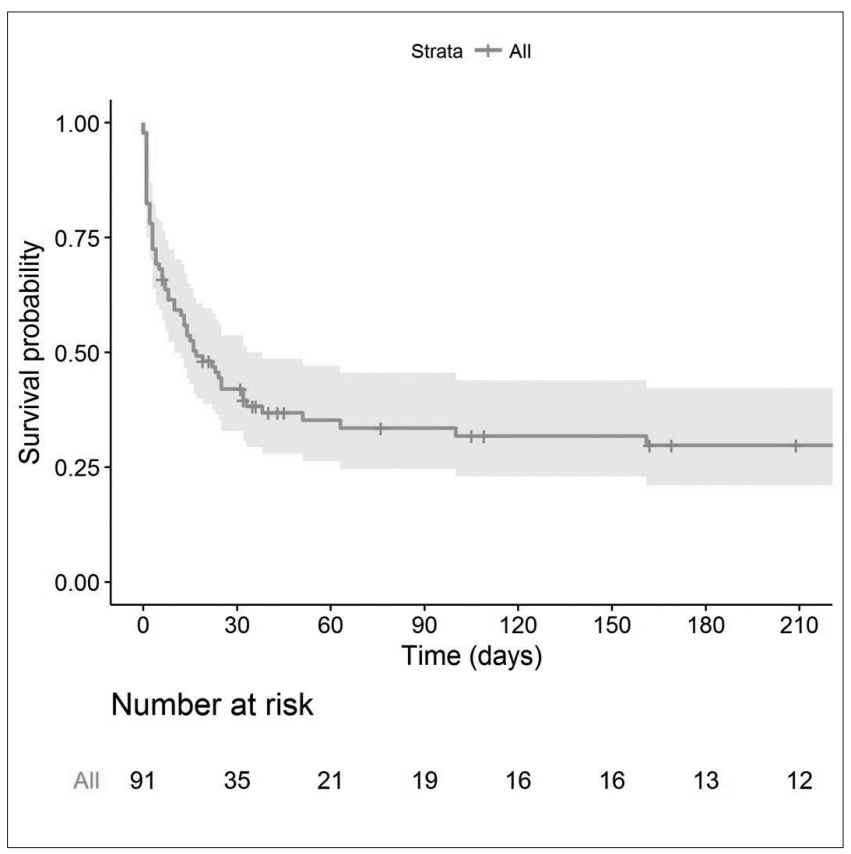

Fig. 1 Kaplan-Meier curve showing overall survival rate of AKI patients treated with SLED

SLED predictors serum creatinine, serum potassium, serum albumin and number of SLEDs were useful in predicting survival. No significant number of haemorrhagic or haemodynamic complications resulting from the SLED treatment occured.

The short survival time of less than 30 days found in our study corroborated with previous studies in Canada and the USA ${ }^{6,8,9}$ but higher than studies from China and Germany. ${ }^{10,11}$ This may be explained by the pre-SLED differences in the severity scores of illness as measured by SOFA, as patients with higher scores had higher mortality rates. However, one randomised trial showed a high 30-day mortality $(83.3 \%)$ even though the average severity score was low ${ }^{12}$, indicating that other factors besides the severity score also influences the mortality rate. In addition, similar to our findings, admission to medical ICU was reported to be associated with mortality after CRRT in a study by Pérez-Fernández et al. ${ }^{13}$ In another study by Abdula et al., survival curve analysis found that a third of the patients died within 1 week of treatment, with a median survival of 15 days. $^{9}$

Pre-SLED predictors of survival were serum albumin, serum creatinine, serum potassium, and number of SLEDs. We found that the higher the pre-SLED serum albumin, the lower the death rate. The reason for this is not entirely clear, a meta analysis found that hypoalbuminemia was a predictor of both AKI and death after AKI development. ${ }^{14}$ Likewise, pre-SLED serum creatinine was a predictor for survival, which may be associated with the number of SLED treatments in patients with high serum creatinine. ${ }^{2,9,14}$ In constrast, high pre-SLED serum potassium increased the risk of death which may be explained by hyperkalemia being associated with cardiovascular problems. ${ }^{15,16}$

Our study found that SLED treatments provided haemodynamic stability and were associated with less serious complications, as other studies have also found. ${ }^{6,7,16,17}$ Recently, 2 systematic reviews indicated that SLED was a safe and effective modality for treating AKI in critically ill patients. ${ }^{18,19}$ The findings of our study can be generalilsed to other settings because the patient age, gender profile, major causes of AKI and comorbid diseases were similar to other studies. ${ }^{6,10}$ Moreover, the details of the SLED treatment in our study in terms of SLED treatments per patient, blood flow rate, dialysate flow rate, duration of SLED treatments, and use of normal saline as an anticoagulant are similar to several previous studies. ${ }^{6,10,20,21}$ It has been suggested that the use of normal saline as a SLED anticoagulant may cause clotting in the SLED circuit leading to blood $\operatorname{loss}^{22}$, but this did not occur in our study. 
Table 4. Clinical responses and complications

\begin{tabular}{lccc}
\hline Variables & Pre-SLED & Post-SLED & $P$-value \\
\hline MAP (mmHg), mean \pm SD & $80.7(15.1)$ & $78.3(15.8)$ & 0.29 \\
\hline Number of vasopressor use & $1.3(0.6)$ & $1.5(0.9)$ & 0.32 \\
Arrhythmia, n (\%) & & $3(3.3)$ & \\
Intradialytic hypotension, $\mathrm{n}(\%)$ & $12(13.2)$ & \\
Bleeding, $\mathrm{n}(\%)$ & & $0(0)$ & \\
\hline
\end{tabular}

MAP: Mean Arterial Pressure

Table 5. Predictors of survival rate of AKI patients treated with SLED by Cox regression analysis

\begin{tabular}{lccc}
\hline Variable & Crude HR $(\mathbf{9 5} \% \mathbf{C I})$ & Adjusted HR $\mathbf{( 9 5 \%}$ CI) & $P$-value \\
\hline Pre-SLED serum creatinine $(\mathrm{mg} / \mathrm{dl})$ & $0.81(0.71,0.9)$ & $0.82(0.71,0.94)$ & 0.002 \\
Pre-SLED serum albumin $(\mathrm{mg} / \mathrm{dl})$ & $0.56(0.39,0.82)$ & $0.57(0.4,0.81)$ & 0.001 \\
Pre-SLED serum potassium $(\mathrm{mg} / \mathrm{dl})$ & $1.21(0.96,1.53)$ & $1.38(1.1,1.73)$ & 0.010 \\
Pre-SLED SOFA & $1.09(1,1.19)$ & $1.08(0.99,1.17)$ & 0.075 \\
Number of SLED (times) & $0.97(0.93,1.02)$ & $0.95(0.91,1)$ & 0.038 \\
\hline
\end{tabular}

AKI: Acute Kidney Injury; SOFA: Sequential Organ Failure Assessment; HR: Hazard Ratio; SLED: Sustained Low Efficiency Dialysis

SLED treatment led to improved acidosis after 48 hours as small molecular toxins are eliminated by diffusion..$^{23,24}$

There are only a few studies that have considered the clinical and laboratory factors associated with survival which included clinically relevant measures of comorbidity, baseline serum creatinine, severity of illness and haemodynamic data, as in our study. There are some limitations to our study. Firstly, the study was conducted in one hospital setting. However, the patients were all from an ICU setting in a tertiary care center, which provides the same high level of care as other hospitals in Thailand, Europe and the USA. Second, this was a retrospective study, thus the duration of SLED depended on the decision of the attending nephrologist. Third, some severity data such as organ failure, AKI staging and the length of stay in the ICU were missing.

In conclusion, our study found that SLED treatment was useful for AKI patients, resulting in less haemorrhagic and haemodynamic complications, and almost half of patients surviving after 30 days. We found that laboratory responses are beneficial to predict a patient's survival. The effects of SLED on survival and benefit of predictors should be assessed in other settings as the SLED procedures in other settings may vary.

\section{Acknowledgment}

The authors would like to thank Professor Tippawan Liabsuetrakul of the Epidemiology Unit, Faculty of Medicine, Prince of Songkla University, Thailand, for editing the manuscript.

\section{REFERENCES}

1. Peters E, Antonelli M, Wittebole X, Nanchal R, François B, Sakr Y, et al. A worldwide multicentre evaluation of the influence of deterioration or improvement of acute kidney injury on clinical outcome in critically ill patients with and without sepsis at ICU admission: results from the intensive care over nations audit. Crit Care 2018;22:188.

2. Kes P, Basić Jukić N. Acute kidney injury in the intensive care unit. Bosn J Basic Med Sci 2010 Suppl 1:S8-12.

3. Tan HK, Hart G. Plasma filtration. Ann Acad Med Singapore 2005;34:615-24.

4. Bellomo R, Ronco C. Continuous renal replacement therapy: continuous blood purification in the intensive care unit. Ann Acad Med Singapore 1998;27:426-9. 
5. Fieghen HE, Friedrich JO, Burns KE, Nisenbaum R, Adhikari NK, Hladunewich MA, et al. The hemodynamic tolerability and feasibility of sustained low efficiency dialysis in the management of critically ill patients with acute kidney injury. BMC Nephrol 2010;11:32.

6. Kitchlu A, Adhikari N, Burns KEA, Friedrich JO, Garg AX, Klein D, et al. Outcomes of sustained low efficiency dialysis versus continuous renal replacement therapy in critically ill adults with acute kidney injury: a cohort study. BMC Nephrol 2015;16:127.

7. Zhang L, Yang J, Eastwood GM, Zhu G, Tanaka A, Bellomo R. Extended daily dialysis versus continuous renal replacement therapy for acute kidney injury: a meta-analysis. Am J Kidney Dis 2015;66:322-30.

8. Edrees F, Li T, Vijayan A. Prolonged intermittent renal replacement therapy. Adv Chronic Kidney Dis 2016;23:195-202.

9. Salahudeen AK, Kumar V, Madan N, Xiao L, Lahoti A, Samuels J, et al. Sustained low efficiency dialysis in the continuous mode (C-SLED): dialysis efficacy, clinical outcomes, and survival predictors in critically ill cancer patients. Clin J Am Soc Nephrol 2009; $4: 1338-46$

10. Zhou J, Yang L, Zhang K, Liu Y, Fu P. Risk factors for the prognosis of acute kidney injury under the acute kidney injury network definition: a retrospective, multicenter study in critically ill patients. Nephrology (Carlton) 2012;17:330-7.

11. Schwenger V, Weigand MA, Hoffmann O, Dikow R, Kihm LP, Seckinger J, et al. Sustained low efficiency dialysis using a single-pass batch system in acute kidney injury-a randomized interventional trial: the renal replacement therapy study in intensive care unit patients. crit care 2012;16:R140.

12. Abe M, Okada K, Suzuki M, Nagura C, Ishihara Y, Fujii Y, et al. Comparison of sustained hemodiafiltration with continuous venovenous hemodiafiltration for the treatment of critically ill patients with acute kidney injury. Artif Organs 2010;34:331-8.

13. Pérez-Fernández X, Sabater-Riera J, Sileanu FE, Vázquez-Reverón J, Ballús-Noguera J, Cárdenas-Campos P. Clinical variables associated with poor outcome from sepsis-associated acute kidney injury and the relationship with timing of initiation of renal replacement therapy. J Crit Care 2017;40:154-60.
14. Wiedermann CJ, Wiedermann W, Joannidis M. Hypoalbuminemia and acute kidney injury: a meta-analysis of observational clinical studies. Intensive Care Med 2010;36:1657-65

15. Lazich I, Bakris GL. Prediction and Management of Hyperkalemia Across the Spectrum of Chronic Kidney Disease. Semin Nephrol 2014;34:333-9.

16. Jain N, Kotla S, Little BB, Weideman RA, Brilakis ES, Reilly RF, et al. Predictors of Hyperkalemia and Death in Patients With Cardiac and Renal Disease. Am J Cardiol 2012;109:1510-3.

17. Caires RA, Abdulkader RCRM, Costa E Silva VT, Ferreira GS, Burdmann EA, Yu L, et al. Sustained low-efficiency extended dialysis (SLED) with single-pass batch system in critically-ill patients with acute kidney injury (AKI). J Nephrol 2016;29:401-9.

18. Nash DM, Przech S, Wald R, O'Reilly D. Systematic review and meta-analysis of renal replacement therapy modalities for acute kidney injury in the intensive care unit. J Crit Care 2017;41:138-44.

19. Kovacs B, Sullivan KJ, Hiremath S, Patel RV. Effect of sustained low efficient dialysis versus continuous renal replacement therapy on renal recovery after acute kidney injury in the intensive care unit: A systematic review and meta-analysis. Nephrology (Carlton) 2017;22:343-53.

20. Sethi SK, Bansal SB, Khare A, Dhaliwal M, Raghunathan V, Wadhwani N, et al. Heparin free dialysis in critically sick children using sustained low efficiency dialysis (SLEDD-f): a new hybrid therapy for dialysis in developing world. PLoS ONE 2018;13:e0195536.

21. Fiaccadori E, Regolisti G, Cademartiri C, Cabassi A, Picetti E, Barbagallo M, et al. Efficacy and safety of a citrate-based protocol for sustained low-efficiency dialysis in AKI using standard dialysis equipment. Clin J Am Soc Nephrol 2013;8:1670-8.

22. Tolwani AJ, Wille KM. Anticoagulation for Continuous Renal Replacement Therapy. Semin Dial 2009;22:141-5.

23. Dichtwald S, Weinbroum AA, Sorkine P, Ekstein MP, Dahan E. Metformin-associated lactic acidosis following acute kidney injury. Efficacious treatment with continuous renal replacement therapy. Diabet Med 2012;29:245-50.

24. Regolisti G, Antoniotti R, Fani F, Greco P, Fiaccadori E. Treatment of metformin intoxication complicated by lactic acidosis and acute kidney injury: the role of prolonged intermittent hemodialysis. Am J Kidney Dis 2017;70:290-6. 